\title{
Refined Shortest Paths (RSP): incorporation of topography in space use estimation from node-based telemetry data
}

Niella, Yuri; Flávio, Hugo; Smoothey, Amy F.; Aarestrup, Kim; Taylor, Matthew D.; Peddemors, Victor M.; Harcourt, Robert

Published in:

Methods in Ecology and Evolution

Link to article, DOI:

10.1111/2041-210X.13484

Publication date:

2020

Document Version

Peer reviewed version

Link back to DTU Orbit

Citation (APA):

Niella, Y., Flávio, H., Smoothey, A. F., Aarestrup, K., Taylor, M. D., Peddemors, V. M., \& Harcourt, R. (2020). Refined Shortest Paths (RSP): incorporation of topography in space use estimation from node-based telemetry data. Methods in Ecology and Evolution, 11(12), 1733-1742. https://doi.org/10.1111/2041-210X.13484

\section{General rights}

Copyright and moral rights for the publications made accessible in the public portal are retained by the authors and/or other copyright owners and it is a condition of accessing publications that users recognise and abide by the legal requirements associated with these rights.

- Users may download and print one copy of any publication from the public portal for the purpose of private study or research.

- You may not further distribute the material or use it for any profit-making activity or commercial gain

- You may freely distribute the URL identifying the publication in the public portal 


\section{Methods in Ecology and Evolution}

MR YURI NIELLA (Orcid ID : 0000-0003-1878-6091)

MR HUGO FLÁVIO (Orcid ID : 0000-0002-5174-1197)

PROFESSOR ROBERT G HARCOURT (Orcid ID : 0000-0003-4666-2934)

Article type : Application

Handling editor: Professor Edward Codling

Refined Shortest Paths (RSP): incorporation of topography in space use estimation from node-based telemetry data

Yuri Niella ${ }^{1}$, Hugo Flávio² ${ }^{2}$ Amy F. Smoothey ${ }^{3}$, Kim Aarestrup ${ }^{2}$, Matthew D. Taylor ${ }^{4}$, Victor M.

Peddemors $^{3}$, Robert Harcourt ${ }^{1}$

${ }^{1}$ Department of Biological Sciences, Macquarie University, North Ryde 2113, Sydney, New

South Wales, Australia

${ }^{2}$ National Institute of Aquatic Resources, Technical University of Denmark, Silkeborg, Denmark

${ }^{3}$ NSW Department of Primary Industries, Fisheries Research, Sydney Institute of Marine Science,

Mosman 2088, Sydney, New South Wales, Australia

${ }^{4}$ Port Stephens Fisheries Institute, New South Wales Department of Primary Industries, Taylors

Beach Road, Taylors Beach, NSW 2316, Australia.

*Correspondence author: yuri.niella@gmail.com

Running headline: RSP: telemetry modelling of aquatic space use

This article has been accepted for publication and undergone full peer review but has not been through the copyediting, typesetting, pagination and proofreading process, which may lead to differences between this version and the Version of Record. Please cite this article as doi: 10.1111/2041-210X.13484

This article is protected by copyright. All rights reserved 


\section{Abstract}

1. Acoustic telemetry enables spatial ecologists to collect movement data from a variety of aquatic species. In estuaries and rivers, accounting for the complex shape of water bodies is challenging. Current methods for analysing utilization distributions are restricted to using the locations of receivers where animals have been detected, which limits the information available to estimate movement paths.

2. We present a new R package (RSP - Refined Shortest Paths) for analysing the movements of animals tracked with acoustic transmitters in environments constrained by landmasses. The method estimates the shortest in-water locations of monitored animals between pairs of detections using least-cost path analysis. It then applies dynamic Brownian Bridge Movement Models to calculate utilization distribution areas. Intra- and inter-specific overlaps in space and time are calculated and can be used, for example, to investigate potential influencing environmental factors.

3. Tracks refined with RSP follow the estuary shape, yielding substantially longer, but more realistic travel distances. Showcased examples demonstrate how RSP can be used to analyse intra- and inter-specific movement patterns; determine similarities in habitat use; identify the environmental conditions responsible for influencing the size of the space use areas; and assess levels of spatial overlap between different species.

4. The RSP toolkit generates more realistic movements of tracked animals than those derived using receiver locations alone. An incidental benefit is its ability to deal with receiver loss, a common problem in acoustic telemetry studies. The analysis can be readily customized to suit different study species, array configurations and habitat shapes.

Keywords: constrained random walk, environmental correlates, least-cost analysis, overlapping areas, utilization distribution.

\section{Introduction}

Traditional studies of fine-scale habitat use in the aquatic environment relied on active telemetry techniques (Gannon et al., 2015; Pastor et al., 2009; Taylor \& Ko, 2011). Active tracking is very laborious, costly and requires certain infrastructure which further limits its application. It allows researchers to monitor only a limited number of individuals, usually over short periods of time. Alternatively, measuring fine-scale movements has been possible using the 
time difference of arrival of acoustic detections within passive acoustic arrays (Espinoza et al., 2011). However, as this method requires overlapping detection ranges and large numbers of receivers, realistically only small geographical areas can be covered. This approach can therefore be ineffective for studying finer-scale movements of wide-ranging animals as these might quickly move out of the covered area. Researchers often deploy non-overlapping arrays to increase spatial coverage, which can still potentially generate thousands of detections (i.e. locations) for tracked individuals (Payne et al., 2013). However, when using non-overlapping arrays, the number of unique positions is limited by the number of receivers.

In aquatic environments, land masses are important barriers that limit the movements of animals, yet few analytical tools are capable of accounting for this constraint when estimating space use (Aspillaga et al., 2019; Wilson et al., 2017). Least-cost path analysis takes into account the landscape properties to estimate the best cost-effective routes between two positions, i.e. start and end points. In habitats constrained by landmasses, this approach is useful for calculating the shortest in-water distances between consecutive acoustic detections. These distances can be then used for obtaining the shortest paths tracked animals need to travel to be detected at these locations.

Translating tracking information into utilization distribution (UD) areas is a commonly applied method in spatial ecology (Kie et al., 2010). This allows for the identification of home range areas and their corresponding regions of higher usage, thus revealing important biological patterns such as seasonal migrations, trends in habitat partitioning and spawning periods (Crossin et al., 2017; Heupel et al., 2019). The dynamic Brownian Bridge Movement Model (dBBMM) accounts for the time differences between sampled positions and their respective accuracy, while using conditional random walks to detect behavioural changes along trajectories for estimating UD (Kranstauber et al., 2012).

In this paper, we describe a new method to analyse the fine-scale movements of animals tracked with acoustic telemetry in environments constrained by landmasses to estimate and model space use exclusively in-water. This approach allows researchers to use standardized UDs and/or frequencies of overlap between the different groups of animals or individuals as the input for further statistical analyses. Here, we show the applicability of this toolkit and use a simulated analysis to demonstrate its capacity to account for randomly losing acoustic receivers from arrays, a common issue for long-term tracking studies in marine and estuarine environments. 


\section{Description of the method}

The method was developed in R (R Core Team, 2020) and is available through the Refined Shortest Paths (RSP) package (https://github.com/YuriNiella/RSP), together with a detailed manual. Tagged animals are pre-assigned to one or multiple groups, depending on the requirements of the study (e.g. different species/sexes/sizes). This allows RSP to calculate intergroup overlaps in space and time. The approach is divided into three main steps: (a) estimation of the shortest paths in-water between consecutive acoustic detections, (b) calculating UDs constrained by the surrounding landmasses using dBBMMs, and (c) calculating overlaps between different biological groups. All RSP functions are listed in Table 1 and the workflow (Fig. 1) is detailed below.

\subsection{Preliminary analysis of raw detection and data preparation}

Raw acoustic telemetry data might include false detections (e.g. originating from noise) or detections from animals which appear to be dead (e.g. repetitive detections at a single location over extended periods). In order to include only the most reliable data, RSP requires the raw detections to be first filtered using the R package actel (Flávio, 2020), which is performed through any of its main functions, i.e. explore(), migration() and residency() (Fig. 1). These functions are interactive and automatically identify unusual data while requiring the user to verify and validate/invalidate detections. Only the valid detections will be used by RSP.

Topographic data is included in RSP by providing a shapefile of the study area delimiting the land contours, also imported using actel. This shapefile is converted to a raster using loadShape() according to a user-defined cell size. Then a transition layer for the study area must be created with the transitionLayer() function. Changing the base raster resolution will lead to differences in the results obtained.

\subsection{Estimating the shortest paths in water}

In complex aquatic habitats (e.g. estuarine, lacustrine, riverine), the uncertainty regarding the possible trajectories of animals tracked is low compared to open environments (e.g. marine), because the former are naturally constrained (e.g. river margins, islands, coastline, etc.). By using a transition layer from the study area delimiting land areas, the $\operatorname{run} R S P()$ function applies a leastcost analysis of constrained random walks to estimate the shortest paths between consecutive acoustic detections. 
The detection ranges of each receiver are considered to attribute location errors for the estimated positions. If not specified, a default range of $500 \mathrm{~m}$ is used (Kessel et al., 2014). Consecutive detections may occur either at the same station (Table 2) or at different stations (Table 3). Intermediary locations are only interpolated if the interval is longer than 10 minutes (defined using the time.step argument). For detections at different stations, new intermediary locations are interpolated if the distance between stations is greater than $250 \mathrm{~m}$ (defined by distance).

The longer it takes for an animal to be detected consecutively in an array the more uncertain the possible trajectories become. By default, unique sporadic detections (e.g. a single detection within a 48-hour period) are automatically excluded. Each block of detections separated by less than 24 hours is considered a track. This parameter can also be customized using the max.time argument (in hours). The total distances travelled during each track can be obtained with getDistances() (Table 4).

For each sequence of interpolated locations, the errors gradually increase when moving away from the first detection, at a rate of $5 \%$ of the distance argument. This default value was chosen based on the cut-off for statistical significance and simply provides an 'inflation coefficient' to be used during space use calculations. The dBBMM accounts for the elapsed time between consecutive positions to calculate space use areas (Kranstauber et al., 2012), therefore, similar location errors might lead to larger or smaller UDs depending on the animal's speed. Alternatively, a user-defined error increase can be set (in metres) using the argument er.ad. The location error reaches its maximum at the mid-point of the space or time elapsed between the first and second detections and starts decreasing again as the second detection approaches (Tables 2-3).

\subsection{Calculating UD areas}

The UDs are calculated with $\operatorname{dyn} B B M M()$, and performed according to the following temporal resolutions: i) group $\mathrm{dBBMM}$ and ii) timeslot dBBMM. For group dBBMM, UDs are calculated at track level for each animal (Table 5). Alternatively, for timeslot dBBMM the UDs are calculated according to fixed temporal intervals by defining a timeframe argument (in hours). This option divides the monitoring period into smaller windows of equal length (starting at midnight on the first day of data) and calculates the dBBMMs within each temporal window, hereafter referred to as timeslots (Tables 6-7). When a limited number of receivers are used, setting a short timeframe will cause the dBBMMs to include only interpolated locations. 
Therefore, we suggest first exploring the output of plotDensities() to decide the best timeframe to use (Fig. 2). The space use areas are obtained for any contour of interest with getAreas(), for both group and timeslot dBBMM. These can be calculated at track (i.e. individual), or group (i.e. for all individuals simultaneously) levels.

\subsection{Calculating the overlaps between biological groups}

The overlaps are retrieved using getOverlaps(), which only works if group areas are provided. Overlaps correspond to the amount of the smallest group area that lies within the largest group area and are returned both in absolute and percentage values. For group dBBMM analysis, overlaps represent the overall amounts of spatial overlap between two groups, whereas when timeslot dBBMM are used, the overlaps are calculated separately for each timeslot.

\section{Testing the method}

\subsection{Intraspecific patterns of space use: RSP performance}

RSP provides realistic tracks of monitored animals following the water contours (Fig. 3). The dBBMMs can be visualized using plotContours() (Fig. 4), and its output reveals individualspecific movement patterns (Fig. 5a), together with temporal variations in space use (Fig. 5b). Comparing the runRSP() output, all individuals have travelled significantly longer distances (paired T-test, $\mathrm{t}=-3.01, \mathrm{df}=11, \mathrm{p}$-value $=0.012$ ) when using the RSP tracks in comparison with only the receiver locations (Fig. 5c). This indicates that, by generating exclusively in-water paths, RSP provides more accurate movement patterns as it accounts for the generally non-linear trajectories between stations. Details on the biological applicability can be found in Appendix 1 .

\subsection{Interspecific patterns of fine-scale space use}

A daily resolution (i.e. timeframe $=24$ hours) was used to obtain standardized UDs (i.e. timeslot $\mathrm{dBBMM}$ ) and the overlaps in space and time for three species. The daily distributions of the $50 \%$ contours (i.e. areas where animals spent half of the tracking time), revealed periods when each species used larger/smaller areas (Fig. 6a). Further statistical analysis identified significant influences of abiotic conditions upon space use and overlap between species (Appendix 1; Fig. 6bc). The plotAreas() function shows the combined space use when multiple individuals are simultaneously detected on a particular timeslot (Fig. 7). Overlapping areas can be plotted using 
plotOverlaps(), which allows to investigate the exact locations where different groups co-occurred (Fig. 8).

\subsection{Loss of individual receivers from an array}

A simulation study showed significant relationships between percentage receiver loss and the total distances travelled, and the sizes of the 50\% and 95\% contour areas (Appendix 1). The inter-individual variations observed (Fig. 9) indicate that results are likely to vary, not only depending on which receivers are lost, but also on the individual-specific movements (Fig. 10). Nevertheless, similar null effect thresholds were observed across all individuals and metrics analysed (Fig. 9), suggesting that RSP can overcome issues with space use calculations if less than $25 \%$ of receivers are lost. However, study-specific elements such as the number of receivers or the deployment locations are likely to influence the threshold of how many receivers can be lost before impacting results.

\section{Conclusions}

Refined Shortest Paths (RSP) is an efficient tool providing realistic estimates of animal space use in environments constrained by landmasses and can be easily customized depending on the array configuration and species monitored. This package not only provides a tool for calculating standardized UDs, but also quantifies the overlaps between the different biological groups monitored. Overall, movements are analysed by calculating overlaps in space, and finescale patterns can be analysed by calculating overlaps in both space and time. The latter might be used for applying further statistical analysis to test hypotheses about the potential spatial, temporal and environmental variables influencing areas of space use and also the co-occurrence of different species or groups of interest (e.g. sex, age/length). The array configuration is likely to influence the potential of RSP to overcome limitations as a consequence of receiver loss. We reinforce that this is not a primary goal from RSP and should be seen as an additional benefit from the analysis.

\section{Acknowledgments}

We are grateful to M. Kristensen for sharing the brown trout data from the Limfjord, Denmark, and to A. Becker, M. Lowry and R. Brownette who contributed to the work in Lake Macquarie. Special thanks to J. Reed and J. Fitzsimmons for their support during the development of the package. Financial support to YVN through an International Macquarie University Research 
Training Program scholarship is deeply acknowledged. Bull shark tagging in Sydney Harbour was done under research permits to AFS via NSW DPI Animal Care and Ethics Committee (Ref. 07/08) and NSW DPI Research Permit Section 37 (PO1/0059A-2.0).

\section{Author's contributions}

YN, HF, VP, AS and RH conceived the ideas and designed the methodology; AS, VP, KA and MT collected the data; YN and HF wrote the package code, analysed the data and led the writing of the manuscript. All authors contributed critically to the drafts and gave final approval for publication.

\section{Data availability}

The package code is stored on GitHub (https://github.com/YuriNiella/RSP) and a Zenodo repository ( https://zenodo.org/record/3981529).

\section{References}

Aspillaga, E., Safi, K., Hereu, B., \& Bartumeus, F. (2019). Modelling the three-dimensional space use of aquatic animals combining topography and Eulerian telemetry data. Methods in Ecology and Evolution, February, 2041-210X.13232. https://doi.org/10.1111/2041210X.13232

Crossin, G. T., Heupel, M. R., Holbrook, C. M., Hussey, N. E., Lowerre-Barbieri, S. K., Nguyen, V. M., Raby, G. D., \& Cooke, S. J. (2017). Acoustic telemetry and fisheries management. Ecological Applications, 27(4), 1031-1049. https://doi.org/10.1002/eap.1533

Espinoza, M., Farrugia, T. J., Webber, D. M., Smith, F., \& Lowe, C. G. (2011). Testing a new acoustic telemetry technique to quantify long-term, fine-scale movements of aquatic animals. Fisheries Research, 108(2-3), 364-371. https://doi.org/10.1016/j.fishres.2011.01.011

Flávio, H. (2020). actel: Acoustic Telemetry Data Analysis (R package version 1.0.0). CRAN. https://cran.r-project.org/package=actel

Gannon, R., Payne, N. L., Suthers, I. M., Gray, C. A., van der Meulen, D. E., \& Taylor, M. D. (2015). Fine-scale movements, site fidelity and habitat use of an estuarine dependent sparid. Environmental Biology of Fishes, 98(6), 1599-1608. https://doi.org/10.1007/s10641-0150385-5

Heupel, M. R., Munroe, S. E. M., Lédée, E. J. I., Chin, A., \& Simpfendorfer, C. A. (2019). 
Interspecific interactions, movement patterns and habitat use in a diverse coastal shark assemblage. Marine Biology, 166(6), 1-17. https://doi.org/10.1007/s00227-019-3511-7

Kessel, S. T., Cooke, S. J., Heupel, M. R., Hussey, N. E., Simpfendorfer, C. A., Vagle, S., \& Fisk, A. T. (2014). A review of detection range testing in aquatic passive acoustic telemetry studies. Reviews in Fish Biology and Fisheries, 24(1), 199-218. https://doi.org/10.1007/s11160-013-9328-4

Kie, J. G., Matthiopoulos, J., Fieberg, J., Powell, R. A., Cagnacci, F., Mitchell, M. S., Gaillard, J. M., \& Moorcroft, P. R. (2010). The home-range concept: Are traditional estimators still relevant with modern telemetry technology? Philosophical Transactions of the Royal Society B: Biological Sciences, 365(1550), 2221-2231. https://doi.org/10.1098/rstb.2010.0093

Kranstauber, B., Kays, R., Lapoint, S. D., Wikelski, M., \& Safi, K. (2012). A dynamic Brownian bridge movement model to estimate utilization distributions for heterogeneous animal movement. Journal of Animal Ecology, 81(4), 738-746. https://doi.org/10.1111/j.13652656.2012.01955.x

Niella, Y., \& Flávio, H. (2020). YuriNiella/RSP: First released version (0.0.1). Zenodo. https://doi.org/10.5281/zenodo.3981529

Pastor, J., Verdoit-Jarraya, M., Astruch, P., Dalias, N., Nelva Pasqual, J. S., Saragoni, G., \& Lenfant, P. (2009). Acoustic telemetry survey of the dusky grouper (Epinephelus marginatus) in the Marine Reserve of Cerbère-Banyuls: informations on the territoriality of this emblematic species. Comptes Rendus - Biologies, 332(8), 732-740. https://doi.org/10.1016/j.crvi.2009.03.010

Payne, N. L., van der Meulen, D. E., Gannon, R., Semmens, J. M., Suthers, I. M., Gray, C. A., \& Taylor, M. D. (2013). Rain reverses diel activity rhythms in an estuarine teleost. Proceedings of the Royal Society B: Biological Sciences, 280(1750). https://doi.org/10.1098/rspb.2012.2363

Taylor, M. D., \& Ko, A. (2011). Monitoring acoustically tagged king prawns Penaeus (Melicertus) plebejus in an estuarine lagoon. Marine Biology, 158(4), 835-844. https://doi.org/10.1007/s00227-010-1610-6

Wilson, K., Hanks, E., \& Johnson, D. (2017). Estimating animal utilization densities using continuous-time Markov chain models. Methods in Ecology and Evolution, 12(10), 32183221. https://doi.org/10.1111/2041-210X.12967 
Table 1. List of RSP analysis and plot functions, and their respective usages including information on the important default arguments.

\begin{tabular}{|c|c|c|c|}
\hline Type & Function & Usage & Important arguments \\
\hline \multirow[t]{5}{*}{ Analysis } & runRSP() & $\begin{array}{l}\text { Estimates the refined shortest paths between } \\
\text { consecutive acoustic detections. }\end{array}$ & $\begin{array}{l}\text { time.step }=10 \text { (minutes); min.time }=10 \\
\text { (minutes); } \text { max.time }=24 \text { (hours); distance }= \\
250 \text { (metres); er.ad }=5 \% \text { of distance (or it } \\
\text { can be set in metres). }\end{array}$ \\
\hline & getDistances() & $\begin{array}{l}\text { Calculates the total distances travelled in each RSP } \\
\text { track, using only the receiver locations and the RSP } \\
\text { interpolated positions. }\end{array}$ & \\
\hline & dynBBMM() & $\begin{array}{l}\text { Calculates dynamic Brownian Bridge Movement } \\
\text { Models (dBBMM) for each tracked animal, both at } \\
\text { track level and also according to standardized fixed } \\
\text { temporal windows (if timeframe is set in hours). }\end{array}$ & $\begin{array}{l}\text { A temporal interval of interest can be set for } \\
\text { analysis using the start.time and stop.time } \\
\text { arguments. }\end{array}$ \\
\hline & getAreas() & $\begin{array}{l}\text { Calculates the areas of space use (in squared } \\
\text { metres) at track or group level, for particular } \\
\text { dBBMM contours of interest. }\end{array}$ & $\begin{array}{l}\text { type }=\text { either "group" or "track" and must be } \\
\text { set; breaks }=50 \% \text { and } 95 \% \text { dBBMM contours } \\
\text { are used. }\end{array}$ \\
\hline & getOverlaps() & $\begin{array}{l}\text { When multiple biological groups are tracked, } \\
\text { calculates the amount of overlap between them. }\end{array}$ & $\begin{array}{l}\text { Overlaps are always calculated for the same } \\
\text { contours obtained using the getAreas() } \\
\text { function. }\end{array}$ \\
\hline
\end{tabular}

This article is protected by copyright. All rights reserved 
Plot

$\begin{array}{ll}\text { plotRaster() } & \text { Verifies that the stations are in the water using the } \\ \text { base raster provided. } & \begin{array}{l}\text { Suggests the most suitable dimensions for saving } \\ \text { the base raster as a projected map. }\end{array} \\ \text { plotTracks() } & \text { Plots a map of an RSP track of interest. } \\ \text { plotDensities() } & \text { Density plot of elapsed time between consecutive } \\ & \text { acoustic detections. Can be either used for all } \\ & \text { monitored animals or for a particular group of } \\ & \text { interest. } \\ \text { plotDistances() } & \text { Plots the total distances travelled by tracked } \\ & \text { animals. }\end{array}$

plotContours() Plots the dBBMM for a particular animal and track of interest.

plotAreas()
Plots the space use areas at group level. land.col $=$ colour of the land masses.

$\max =$ desired size of the longest plot edge.

land.col $=$ colour of the land masses

group: character vector defining the group of interest. If not set, will return the distribution for all monitored animals.

by.group $=$ FALSE (returns a single plot for all tracked animals); compare $=$ TRUE

(distances are returned both using only receiver locations and the RSP interpolations).

breaks $=$ plots the $25 \%, 50 \%, 75 \%$ and $95 \%$ contours; land.col $=$ colour of the land masses.

timeslot must be set for timeslot $\mathrm{dBBMM}$; land.col $=$ colour of the land masses. 
plotOverlaps() Plots the overlapping areas between two different groups.

addStations()
Adds the station locations to an RSP plot. timeslot must be set for timeslot dBBMM; land.col $=$ colour of the land masses.

Customization parameters available. 
Table 2. Example of the interpolated locations from the runRSP() output (Position $=$ RSP) when consecutive acoustic detections $($ Position $=$ Receiver) occur on the same receivers. Some columns were omitted for simplification.

\begin{tabular}{llllllll}
\hline Timestamp & Receiver & Transmitter & Error & Longitude & Latitude & Position & Track \\
\hline 2018-03-07 02:05:47 & 115409 & R64K-4075 & 500 & 9.380 & 56.572 & Receiver & Track_03 \\
2018-03-07 02:30:37 & NA & R64K-4075 & 525 & 9.380 & 56.572 & RSP & Track_03 \\
2018-03-07 02:55:27 & NA & R64K-4075 & 550 & 9.380 & 56.572 & RSP & Track_03 \\
2018-03-07 03:20:18 & NA & R64K-4075 & 550 & 9.380 & 56.572 & RSP & Track_03 \\
2018-03-07 03:45:08 & NA & R64K-4075 & 525 & 9.380 & 56.572 & RSP & Track_03 \\
2018-03-07 04:09:59 & 115409 & R64K-4075 & 500 & 9.380 & 56.572 & Receiver & Track_03 \\
\hline
\end{tabular}

This article is protected by copyright. All rights reserved 
Table 3. Example of the interpolated locations from the runRSP() output (Position $=\mathrm{RSP}$ ) when consecutive acoustic detections (Position $=$ Receiver) occur on different receivers. Some columns were omitted for simplification.

\begin{tabular}{llllllll}
\hline Timestamp & Receiver & Transmitter & Error & Longitude & Latitude & Position & Track \\
\hline 2018-04-27 05:27:10 & 100474 & R64K-4125 & 500 & 9.922 & 57.056 & Receiver & Track_05 \\
2018-04-27 05:35:17 & NA & R64K-4125 & 525 & 9.928 & 57.054 & RSP & Track_05 \\
2018-04-27 05:43:24 & NA & R64K-4125 & 550 & 9.935 & 57.053 & RSP & Track_05 \\
2018-04-27 05:51:32 & NA & R64K-4125 & 575 & 9.943 & 57.054 & RSP & Track_05 \\
2018-04-27 05:59:39 & NA & R64K-4125 & 600 & 9.949 & 57.056 & RSP & Track_05 \\
$2018-04-2706: 07: 47$ & NA & R64K-4125 & 625 & 9.955 & 57.058 & RSP & Track_05 \\
$2018-04-2706: 15: 54$ & NA & R64K-4125 & 650 & 9.960 & 57.061 & RSP & Track_05 \\
$2018-04-2706: 24: 01$ & NA & R64K-4125 & 625 & 9.964 & 57.065 & RSP & Track_05 \\
$2018-04-2706: 32: 09$ & NA & R64K-4125 & 600 & 9.968 & 57.068 & RSP & Track_05 \\
2018-04-27 06:40:16 & NA & R64K-4125 & 575 & 9.975 & 57.070 & RSP & Track_05 \\
$2018-04-2706: 48: 24$ & NA & R64K-4125 & 550 & 9.981 & 57.072 & RSP & Track_05 \\
$2018-04-2706: 56: 31$ & NA & R64K-4125 & 525 & 9.986 & 57.074 & RSP & Track_05 \\
$2018-04-2707: 04: 39$ & 107527 & R64K-4125 & 500 & 9.992 & 57.076 & Receiver & Track_05 \\
\hline
\end{tabular}


Table 4. Example of getDistances() output showing the corresponding distances travelled

(Dist.travel) in metres.

\begin{tabular}{llllll}
\hline Animal.tracked & Track & Day.n & Loc.type & Dist.travel & Group \\
\hline R64K-4125 & Track_02 & 3 & Receiver & 14107 & B \\
R64K-4125 & Track_02 & 3 & RSP & 17399 & B \\
R64K-4125 & Track_03 & 4 & Receiver & 182882 & B \\
R64K-4125 & Track_03 & 4 & RSP & 206815 & B \\
R64K-4125 & Track_04 & 2 & Receiver & 25471 & B \\
R64K-4125 & Track_04 & 2 & RSP & 29845 & B \\
\hline
\end{tabular}

Table 5. Metadata examples on the dynamic Brownian Bridge Movement Models calculated for group dBBMM with $d y n B B M M()$. Included are the corresponding number of valid detections (valid.n), first and last detection times (First.time and Last.time, respectively), and track timespan.

\begin{tabular}{lllllll}
\hline Group & Tag & Track & valid.n & First.time & Last.time & Timespan \\
\hline A & R64K-4075 & Track_09 & 303 & $2018-04-24$ & $2018-04-26$ & 37.3 hours \\
& & & & $11: 40: 56$ & $01: 00: 13$ & \\
B & R64K-4125 & Track_03 & 1003 & $2018-04-25$ & $2018-04-28$ & 74.4 hours \\
& & & & $11: 44: 05$ & $14: 10: 14$ & \\
C & R64K-4128 & Track_06 & 146 & $2018-04-28$ & $2018-04-29$ & 22.9 hours \\
& & & & $16: 29: 07$ & $15: 25: 16$ & \\
D & \multirow{2}{*}{ R64K-4138 } & Track_10 & 584 & $2018-07-14$ & $2018-07-18$ & \\
& & & & $22: 10: 35$ & $18: 33: 43$ & 92.4 hours \\
\hline
\end{tabular}

Table 6. Example of a timeslots object saved for a timeslot dBBMM using the $\operatorname{dyn} B B M M()$ function with a daily resolution (timeframe $=24$ hours).

\begin{tabular}{lll}
\hline slot & start & stop \\
\hline 1 & $2013-09-0200: 00: 00$ & $2013-09-0223: 59: 59$ \\
2 & $2013-09-0300: 00: 00$ & $2013-09-0323: 59: 59$ \\
3 & $2013-09-0400: 00: 00$ & $2013-09-0423: 59: 59$ \\
4 & $2013-09-0500: 00: 00$ & $2013-09-0523: 59: 59$ \\
\hline
\end{tabular}

This article is protected by copyright. All rights reserved 
Table 7. Metadata examples on the dynamic Brownian Bridge Movement Models calculated for timeslot $\mathrm{dBBMM}$ with $d y n B B M M()$. Included are the corresponding timeslot (slot), number of valid detections (valid.n), first (First.time) and last detection (Last.time) times, and track timespan.

\begin{tabular}{llllllll}
\hline Group & Tag & Track & slot & valid.n & First.time & Last.time & Timespan \\
\hline Bream & A69-9002-10473 & Track_02 & 261 & 17 & $2014-05-20$ & $2014-05-20$ & 1.8 hours \\
& & & & & $22: 11: 44$ & $23: 58: 35$ & \\
Bream & A69-9002-10473 & Track_03 & 266 & 45 & $2014-05-25$ & $2014-05-25$ & 10.1 hours \\
& & & & & $13: 32: 44$ & $23: 40: 06$ & \\
Luderick & A69-9002-10481 & Track_01 & 243 & 42 & $2014-05-02$ & $2014-05-02$ & 10.7 hours \\
& & & & & $12: 49: 50$ & $23: 32: 07$ & \\
Luderick & A69-9002-10481 & Track_01 & 244 & 21 & $2014-05-03$ & $2014-05-03$ & 19.7 hours \\
& & & & & $00: 37: 42$ & $20: 20: 09$ & \\
Tarwhine & A69-9004-489 & Track_03 & 429 & 86 & $2014-11-01$ & $2014-11-01$ & 17.1 hours \\
& & & & & $02: 21: 36$ & $19: 25: 42$ & \\
Tarwhine & \multirow{2}{*}{ A69-9004-489 } & Track_03 & 430 & 123 & $2014-11-02$ & $2014-11-02$ & 13.4 hours \\
& & & & & $05: 54: 37$ & $19: 18: 01$ & \\
\hline
\end{tabular}


Preliminary analysis and data preparation

\section{suggestSize()}
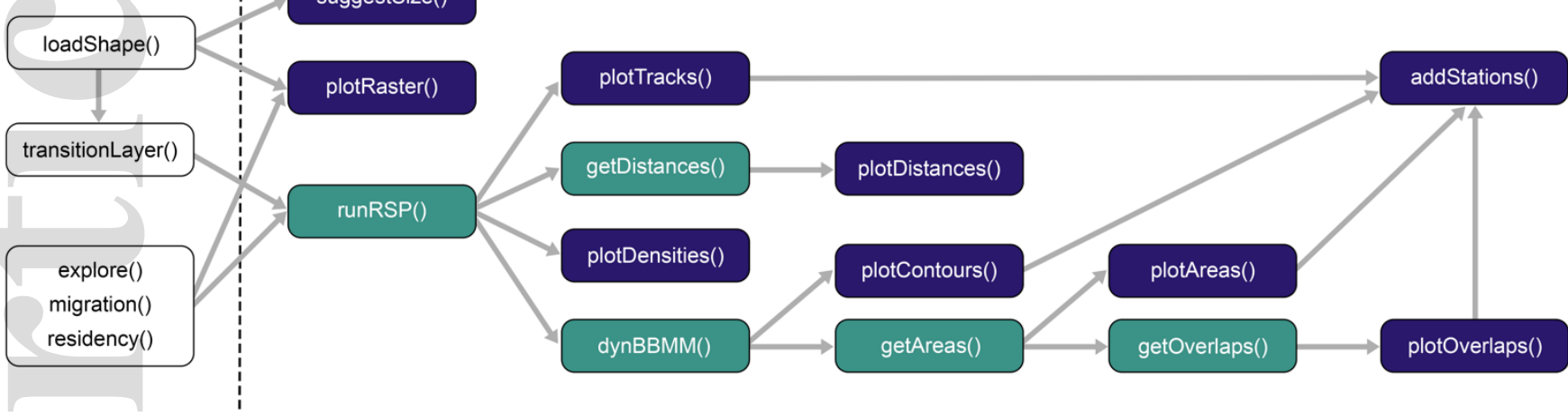

Figure 1. RSP workflow showing the preliminary analysis and data preparation steps using the R package actel.
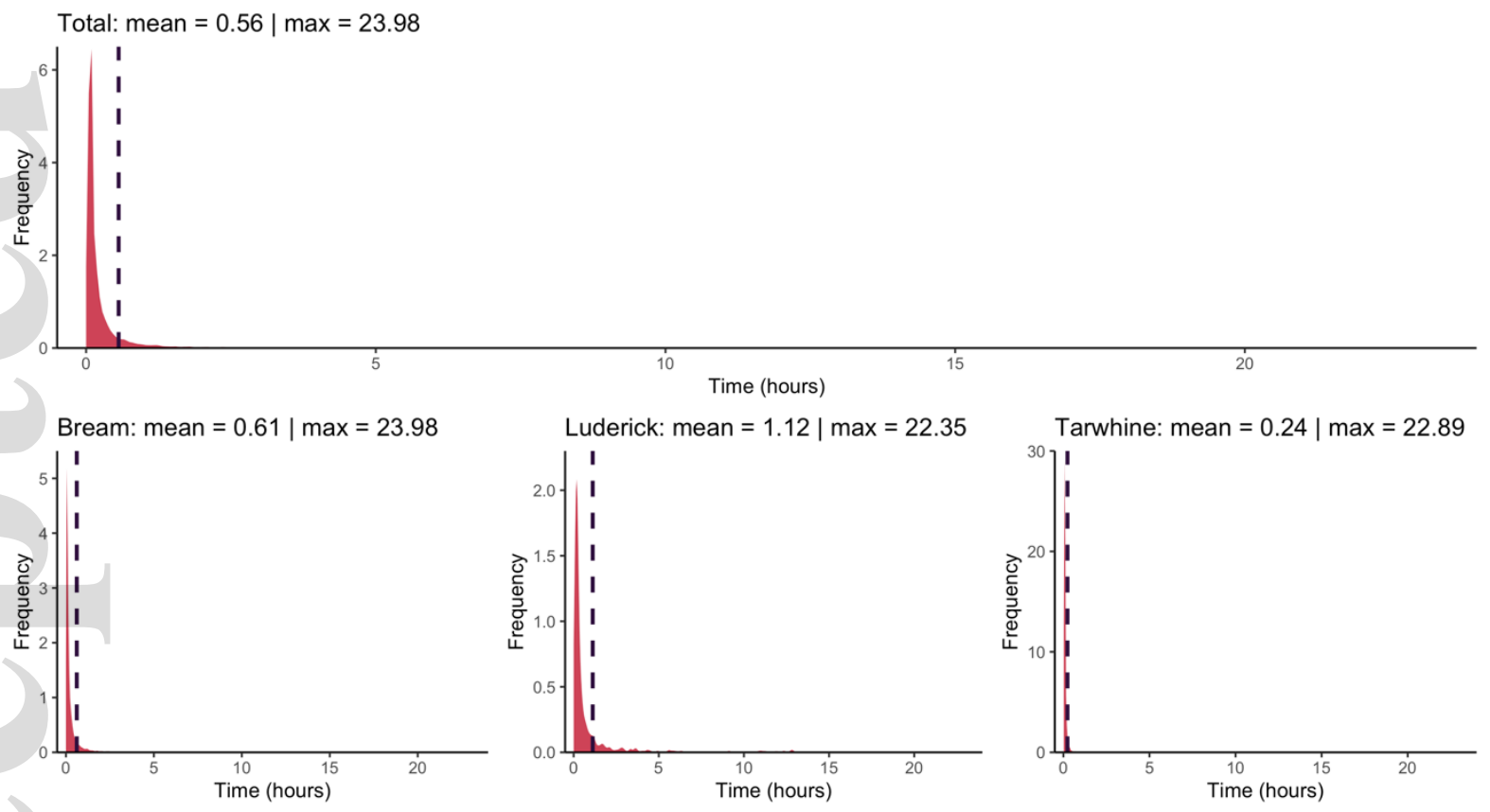

Figure 2. The plotDensities() function can be used to inspect the distributions of elapsed time between consecutive acoustic detections. 
A Track_07

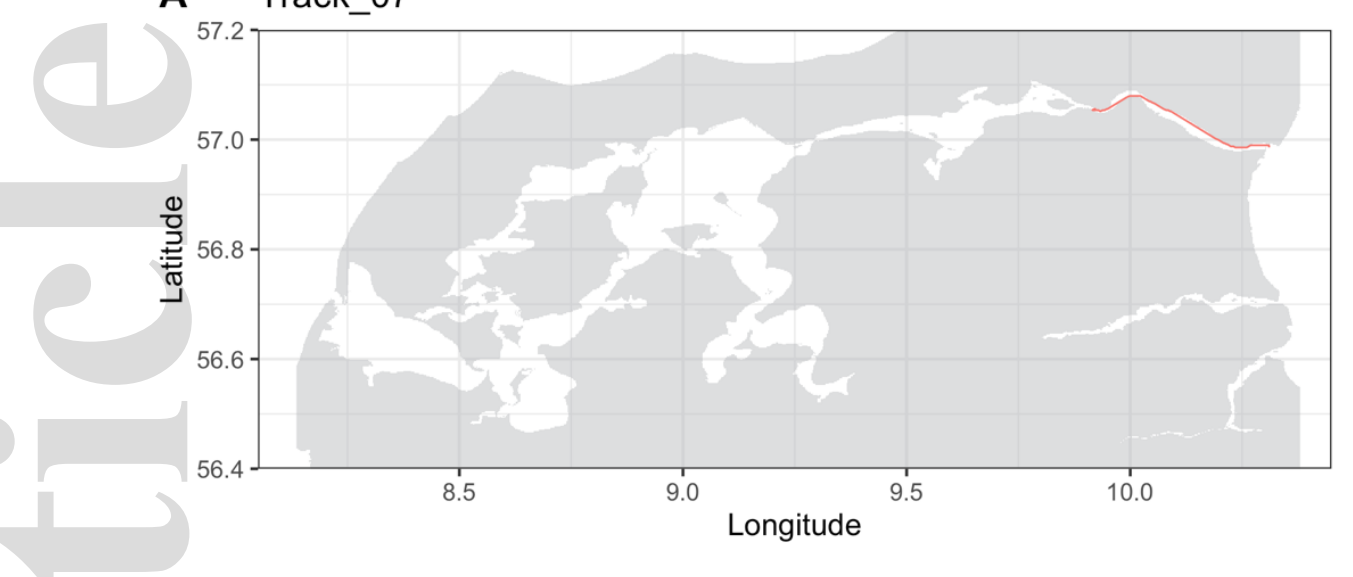

Transmitter - R64K-4128

B Track_09

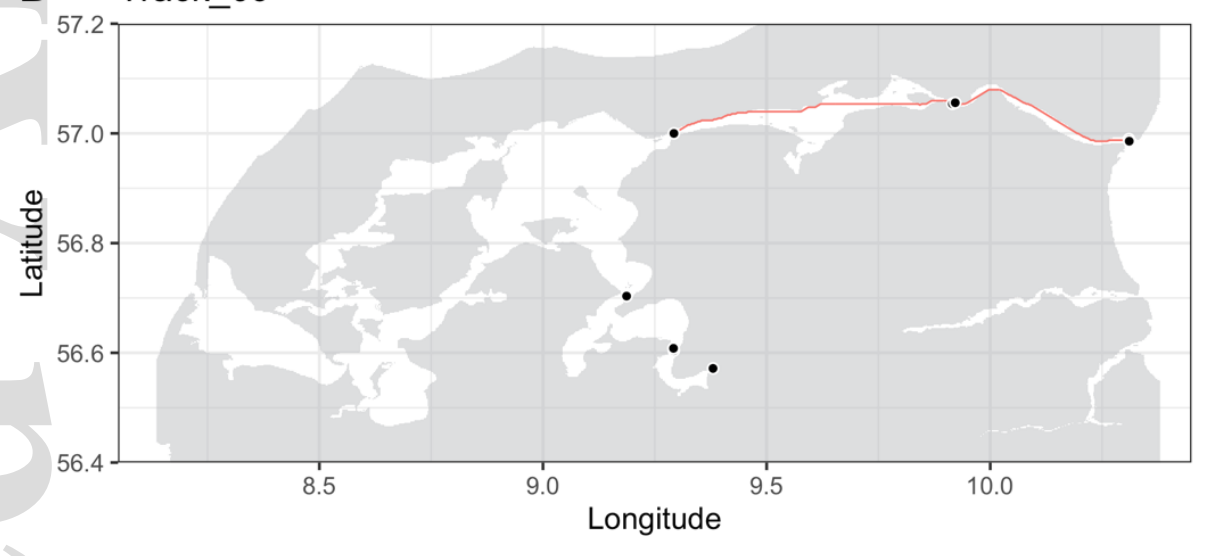

Transmitter - R64K-4075

Figure 3. Example of tracks from runRSP() plotted using plotTracks() (A). Receiver locations can be added to the plot (B) using addStations(). 
A R64K-4138 - Track_10

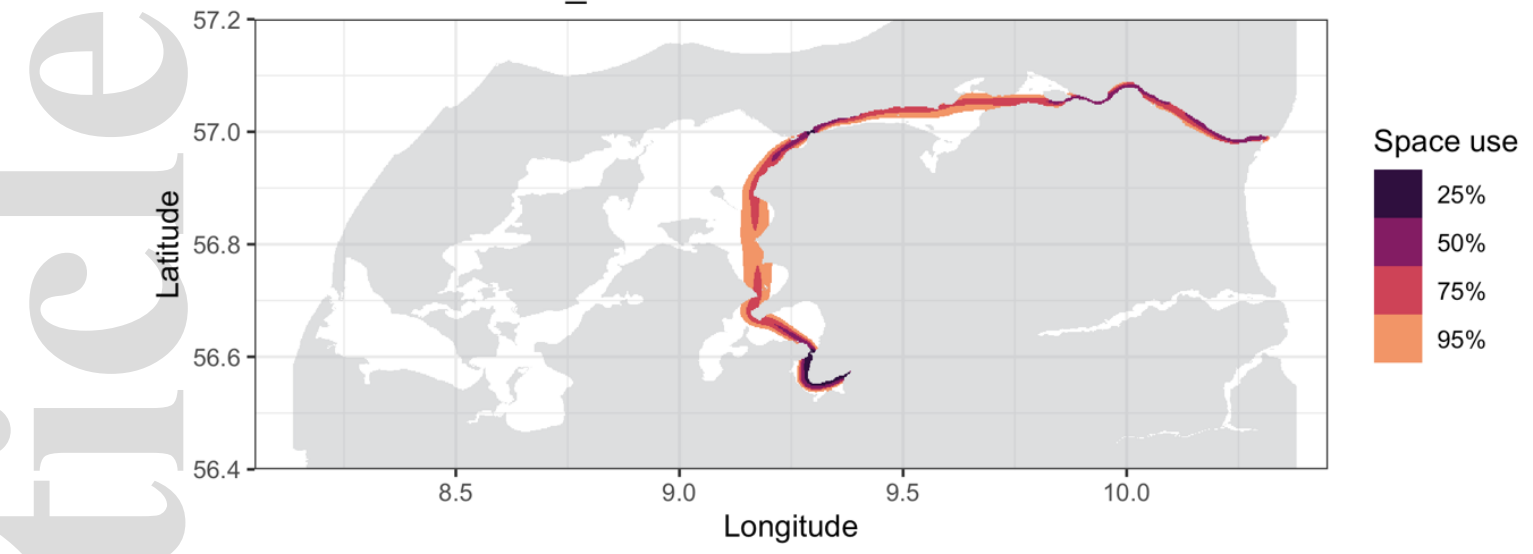

B R64K-4125 - Track_03

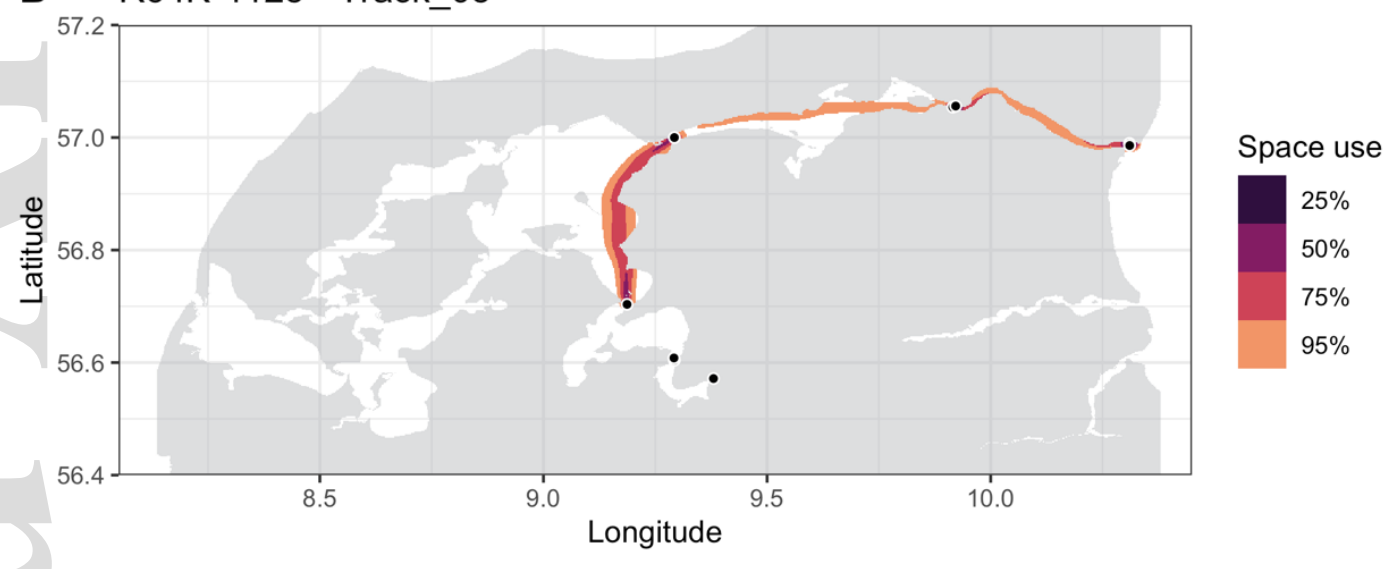

Figure 4. Dynamic Brownian Bridge Movement Models (Table 3) of two brown trout tracked in the Limfjord, Denmark, plotted using plotContours(). 
A

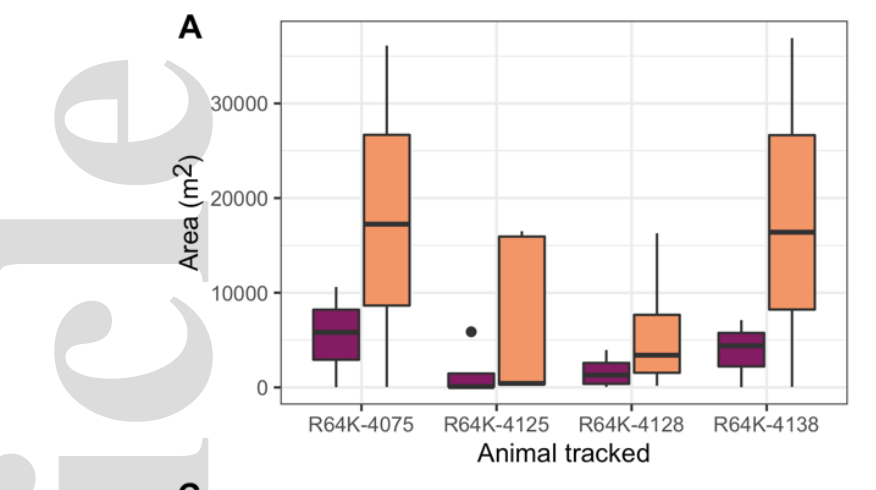

B

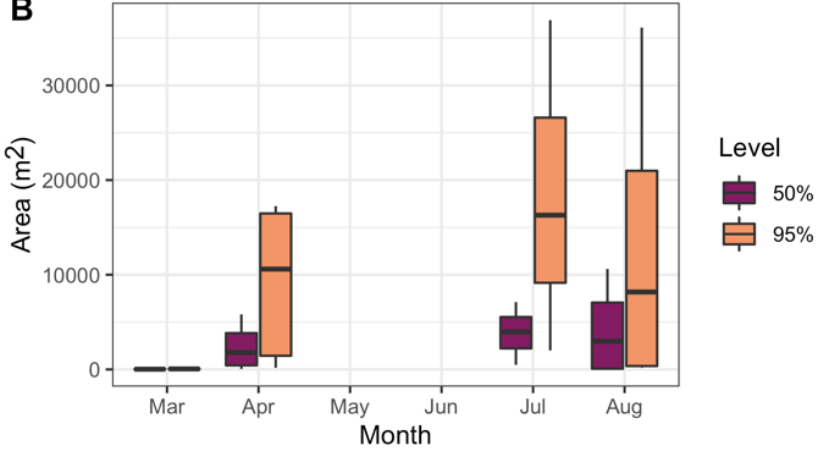

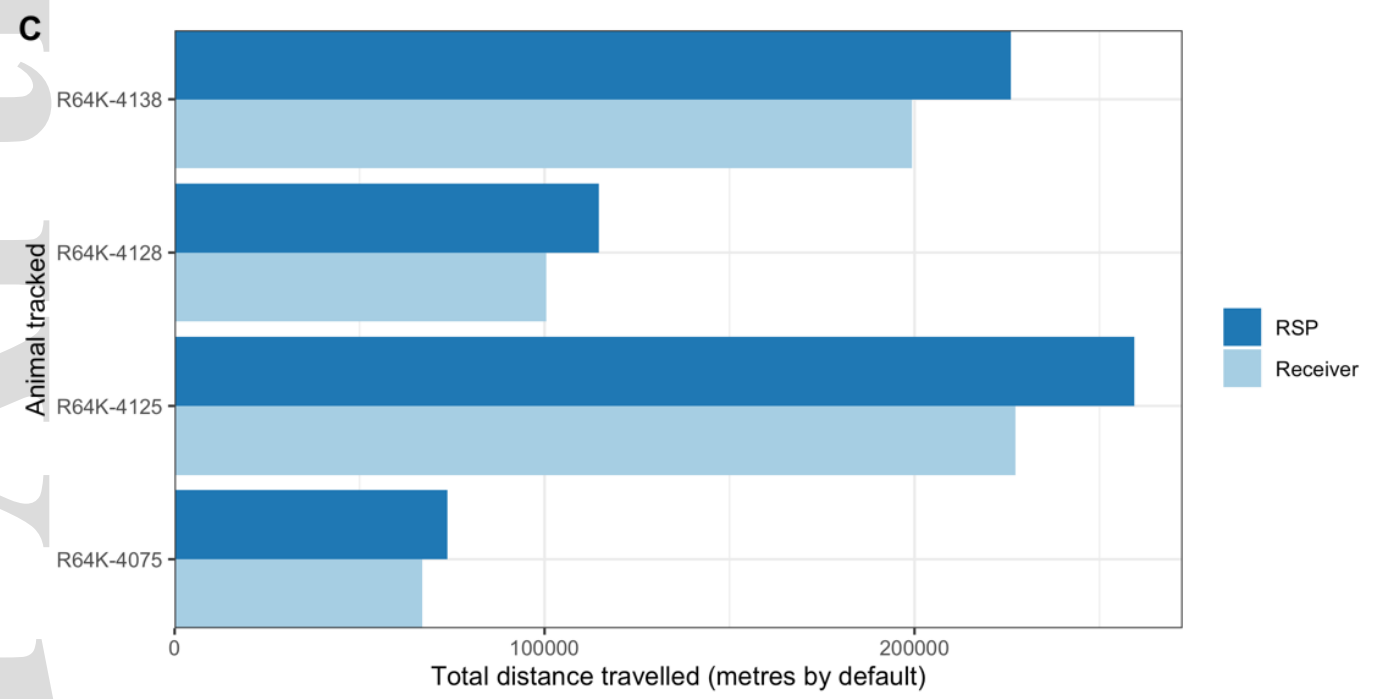

Figure 5. Distribution of space use areas from the dBBMM by (A) animal tracked and (B) month, and (C) total distances travelled calculated with RSP versus only the receiver positions (Receiver) plotted with plotDistances(). 

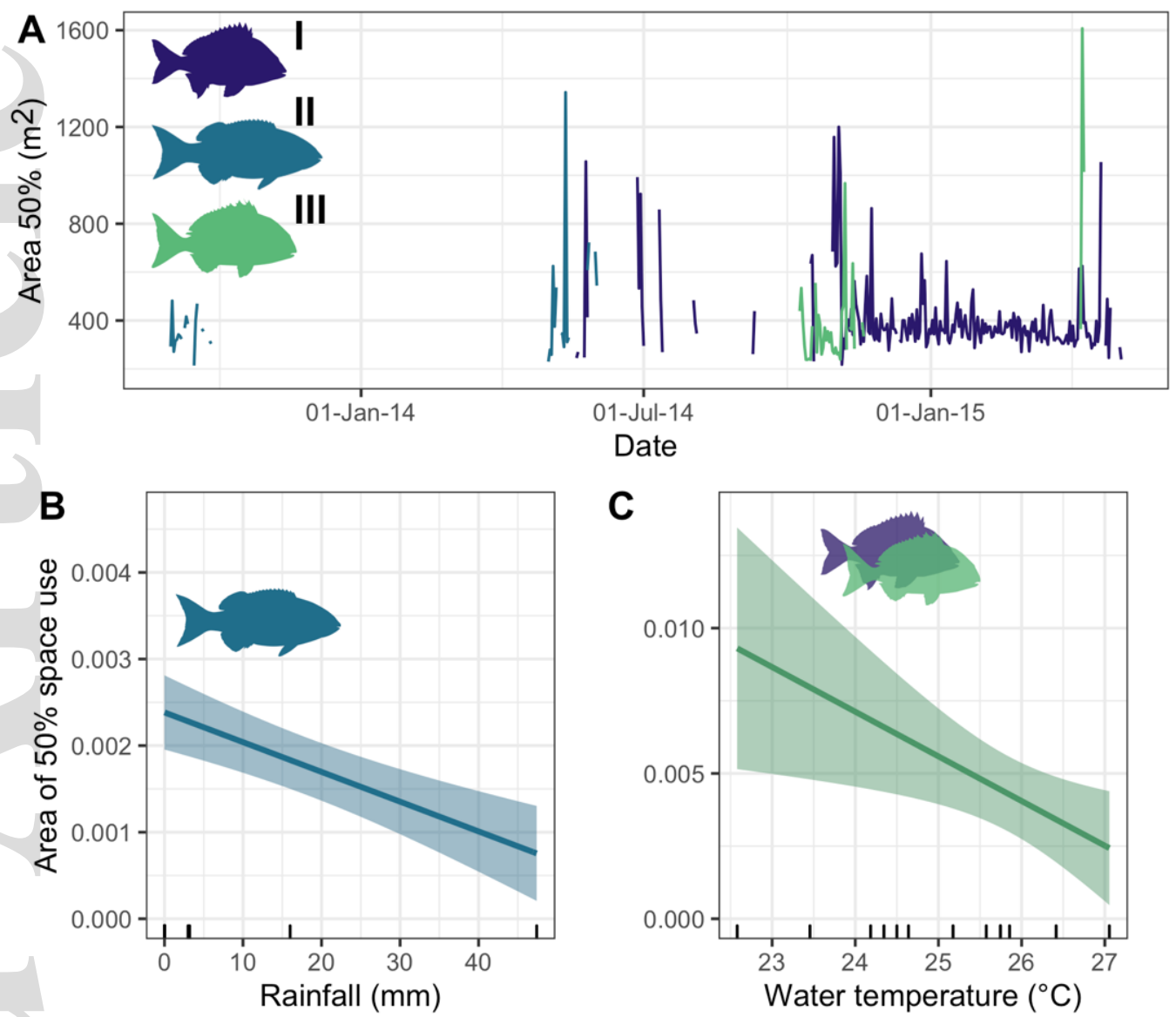

Figure 6. Distributions of (A) daily areas of 50\% use for I = yellowfin bream, II = luderick and III $=$ tarwhine, tracked in Lake Macquarie, Australia. Generalized linear models (Appendix 1) including the effects of (B) rainfall upon the areas of 50\% use of luderick, and (C) water temperature upon the overlaps between yellowfin bream and tarwhine. Shaded areas and dashes (B-C) represent the $95 \%$ confidence intervals and the raw data, respectively. 

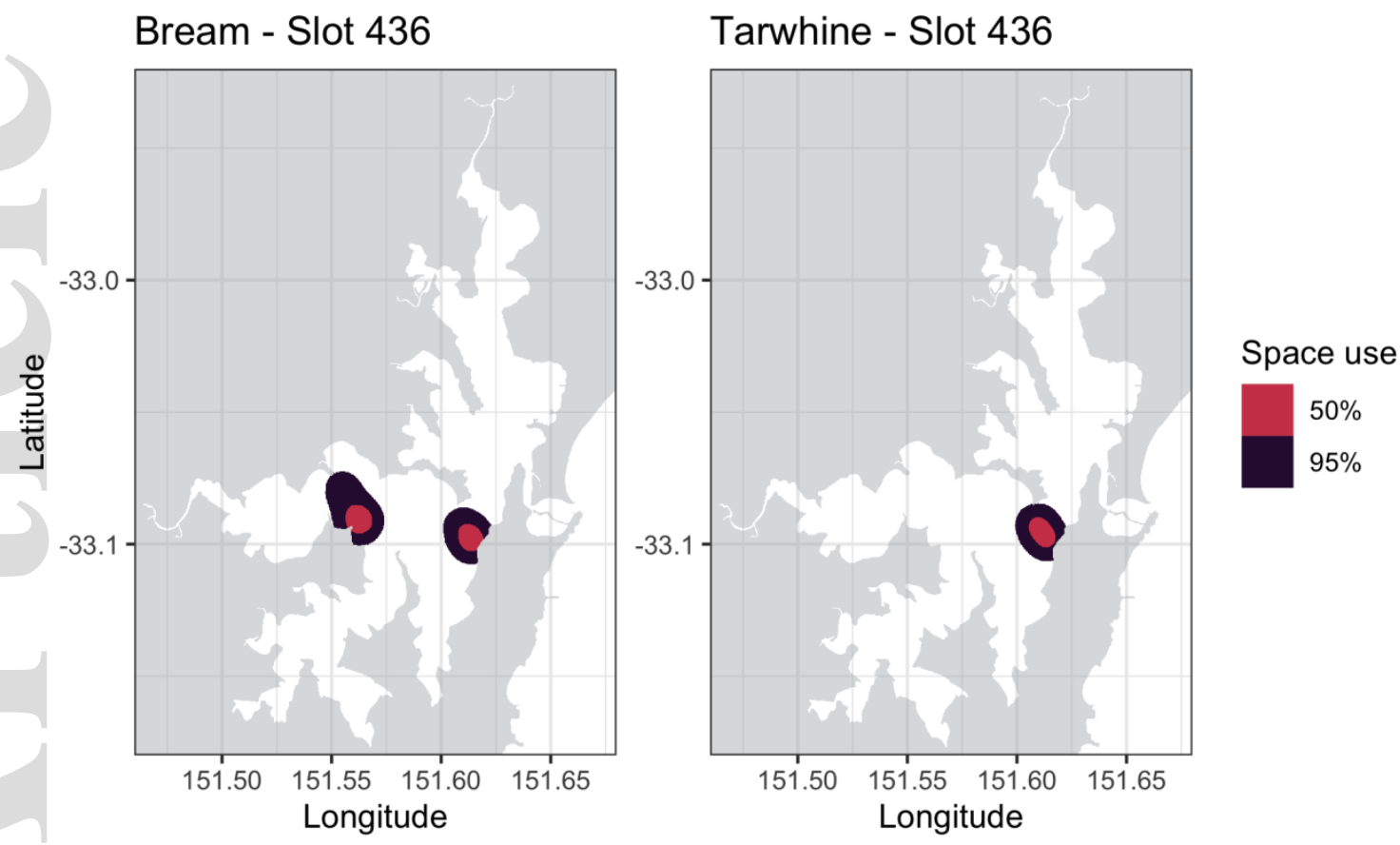

(B)

\begin{tabular}{lllllll}
\hline Group & Tag & Slot & valid.n & First.time & Last.time & Timespan \\
\hline Bream & A69-9004-485 & 436 & 22 & $2014-11-11$ & $2014-11-11$ & 12.0 hours \\
& & & & $11: 09: 26$ & $23: 11: 5412.0$ & \\
\multirow{2}{*}{ Bream } & A69-9004-494 & 436 & 48 & $2014-11-11$ & $2014-11-11$ & 22.6 hours \\
& & & & $00: 53: 57$ & $23: 28: 10$ & \\
Bream & A69-9004-496 & 436 & \multirow{2}{*}{272} & $2014-11-11$ & $2014-11-11$ & 23.9 hours \\
& & & & $00: 05: 57$ & $23: 59: 39$ & \\
Tarwhine & A69-9004-481 & 436 & \multirow{2}{*}{18} & $2014-11-11$ & $2014-11-11$ & 18.2 hours \\
& & & & $05: 11: 40$ & $23: 21: 19$ & \\
& & & & & &
\end{tabular}

Figure 7. Space use areas at group level using plotAreas() for three yellowfin bream and one tarwhine detected during the same timeslot at Lake Macquarie, Australia, including the (A) plots using plotAreas() and (B) the dynBBMM() metadata.

(A) 

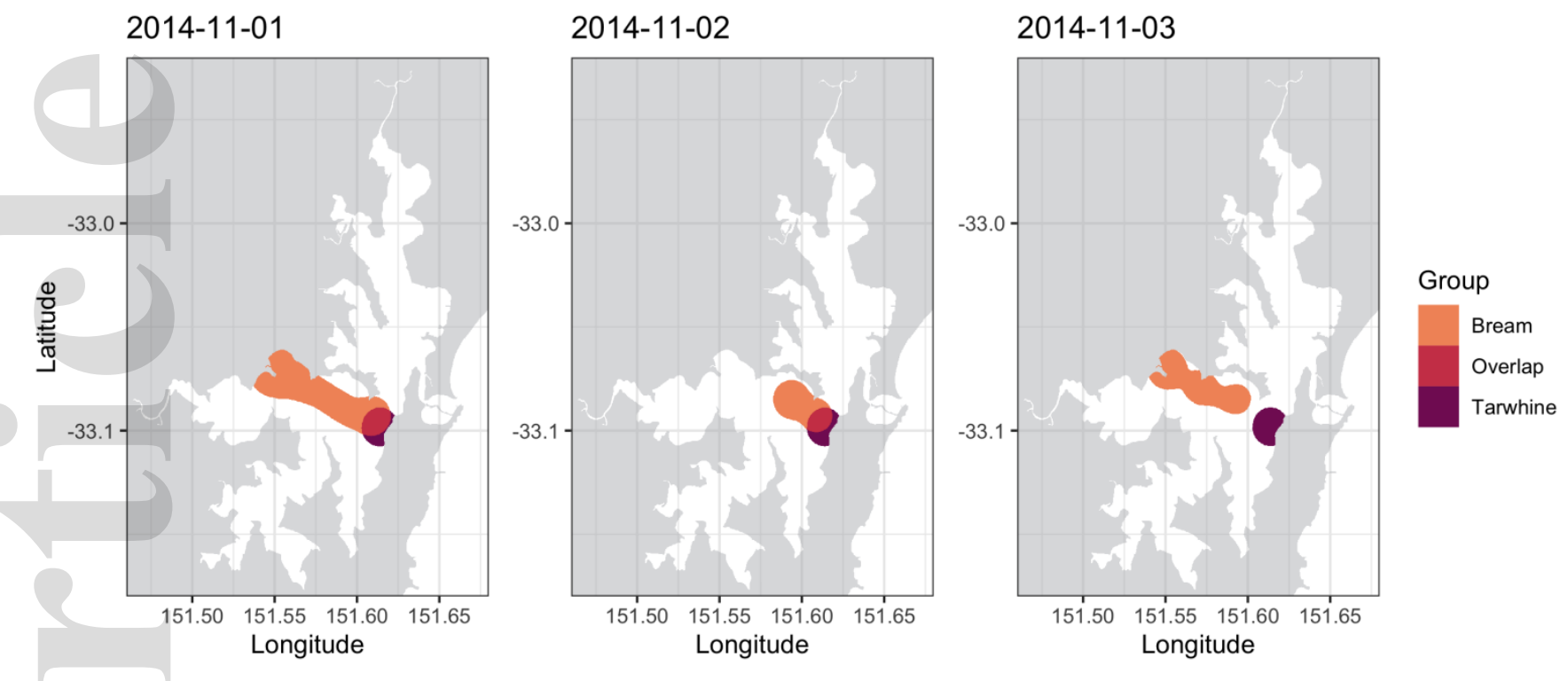

(B)

\begin{tabular}{lllll}
\hline Date & Absolute overlap & Percentage overlap & Bream area & Tarwhine area \\
\hline $2014-11-01$ & 1798386 & 69.5 & 14169558 & 2585492 \\
$2014-11-02$ & 1681317 & 66.1 & 5981752 & 2543978 \\
$2014-11-03$ & 0 & 0 & 8455572 & 2542317
\end{tabular}

Figure 8. Daily areas and overlaps between yellowfin bream and tarwhine at the $95 \%$ contour including (A) the plots with plotOverlaps() and (B) overlaps and areas used in Lake Macquarie, Australia.
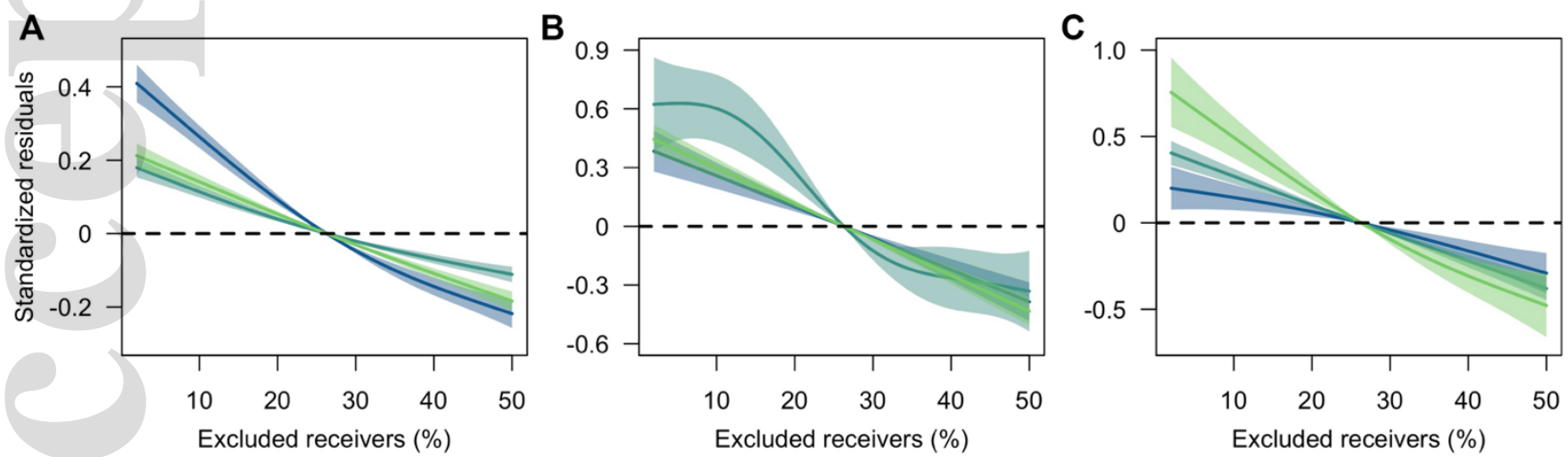

Figure 9. Generalized additive models (Appendix 1) of (A) total distances travelled, (B) 50\% areas and (C) $95 \%$ areas in relation to the frequencies of excluded acoustic receivers for bull 
sharks tracked in Sydney, Australia. Dashed lines and shaded areas represent the null effects and the $95 \%$ confidence intervals, respectively.
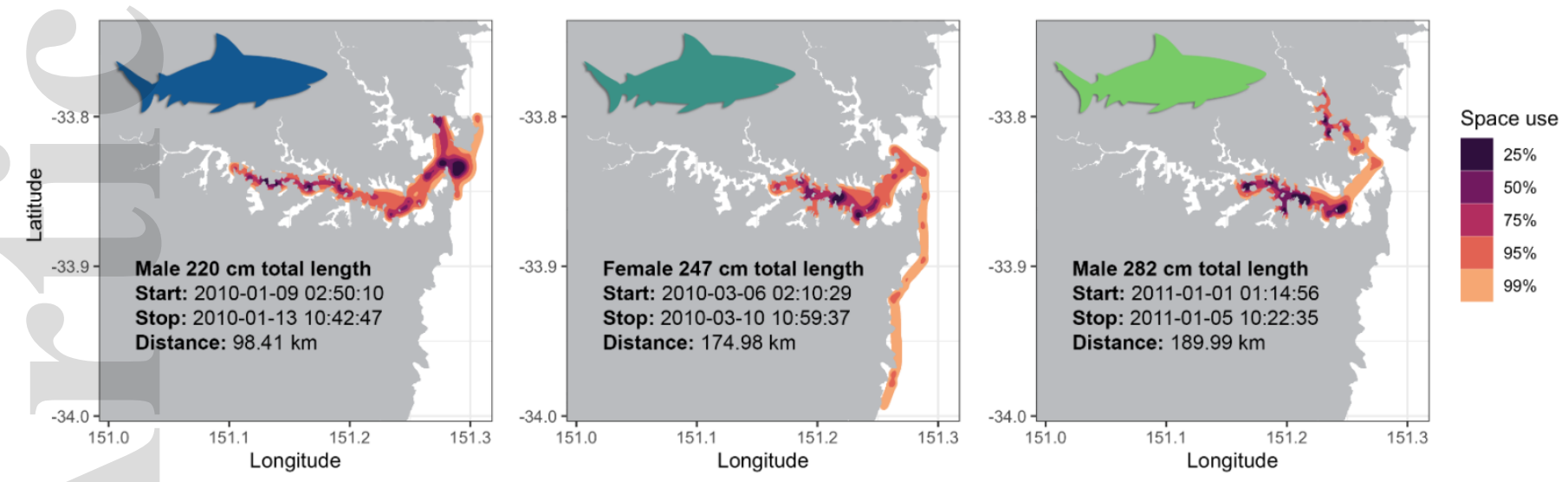

Figure 10. dBBMMs of three bull sharks tracked in Sydney, Australia, on five consecutive days. The colour and transparency of the landmasses can be changed using the argument land.col in plotContours(). 